\title{
COACHING: UNA PSEUDOPSICOLOGÍA DE LA POSMODERNIDAD
}

\section{Coaching: Postmodernity's pseudo psychology}

\author{
Alejandro Khaled Salomón Paredes** \\ Universidad Femenina del Sagrado Corazón
}

\begin{abstract}
Resumen
Con este artículo se busca contribuir en la comprensión del fenómeno coaching, el cual desde hace varios años viene siendo una alternativa de crecimiento personal que presenta numerosas ambigüedades y límites difusos, tanto respecto a su objeto de estudio y trabajo como en su metodología, así como en los requisitos personales y profesionales para su práctica, lo cual representa un serio peligro para la salud psicológica de quienes acuden en busca de ayuda. Las condiciones en las que se gesta y crece el coaching son analizadas dentro de su contexto socio-cultural-económico caracterizado por una marcada tendencia hacia el éxito y la felicidad, con una fuerte orientación facilista. Finalmente se intenta delinear el esbozo de una cultura psicohigiénica que invite a ver la psicología y la psicoterapia más allá de la psicopatología y el modelo médico reduccionista, y orientada más bien hacia una visión integradora de la existencia humana, dedicada no solo a la rehabilitación, sino a la promoción y el desarrollo de las funciones psicológicas y a la promoción de una vida más libre y con más sentido.
\end{abstract}

Palabras clave: Coaching, Programación Neuro Lingüística, pseudopsicología, posmodernidad, psicología, psicoterapia.

\begin{abstract}
This article seeks to contribute to the understanding of the coaching phenomenon, which, for several years, has been an alternative of personal growth that presents numerous ambiguities and diffuse limits, both in its study and work object and in its methodology, as well as in the personal and professional requirements for their practice, which represents a serious danger to psychological health of those who come for help. The conditions in which coaching is produced and developed are analyzed within its socio-cultural-economic context characterized by a marked tendency towards success and happiness, with a strong facilitative orientation. Finally, it attempts to outline a psycho-hygienic culture that invites to see psychology and psychotherapy beyond psychopathology and the reductionist medical model, and oriented more towards a more integrating vision of human existence, dedicated not only to rehabilitation, but to the promotion and development of psychological functions and the promotion of a freer and more meaningful life.
\end{abstract}

Keywords: Coaching, Neuro Linguistic Programming, pseudopsychology, postmodernity, psychology, psychotherapy.

\footnotetext{
* Psicólogo. Docente de la Universidad Femenina del Sagrado Corazón. khaledpsi@ hotmail.com
} 
Cada vez llegan más personas a mi consulta con una problemática común: refieren una mejoría en forma de alivio, empoderamiento o esperanza temporal que luego deriva en un malestar significativo en términos psicológicos y emocionales, tras haber recurrido a un "coach" por alguna razón específica. La experiencia suele ser inicialmente muy auspiciosa: cambios anímicos favorables, una motivación inmensa a lograr todo lo que se propongan y una perspectiva positiva de las circunstancias. Sin embargo tras un leve lapso de tiempo refieren sentirse peor que antes, como si todo lo trabajado no hubiera calado en el ser mismo a profundidad, como si el cambio fuera solo superficial. Estas experiencias con diversas personas me llevaron a pensar en qué factores influyen para que esta práctica de ayuda no estuviese consiguiendo los resultados que las personas buscan cuando se introducen en estos procesos de cambio, o los resultados que el mismo coaching ofrece, incluso llegando a generar daños psicológicos y perturbaciones emocionales. Pero para empezar esta clarificación considero útil definir de qué se trata esta práctica.

\section{¿Qué es el coaching?}

El concepto de coaching surge en el ámbito deportivo, entendiendo al "coach" (palabra de origen inglés que corresponde a entrenador, la cual deriva de carruaje o el que conduce) como aquel que busca orientar o guiar a otros al logro de sus objetivos. Esta palabra se ha extendido a los ámbitos educacional, empresarial y organizacional (Wolk, 2003), e incluso ha llegado al ámbito del crecimiento personal por medio del trabajo psicológico. Es precisamente de este tipo de coaching del que nos ocuparemos, ya que representa la mayor posibilidad de afectación a la salud de las personas. A esta forma de coaching la encontramos en el mercado actual bajo diversos nombres como "coaching con C", "coaching de vida" (life coaching), "coaching transformacional", "coaching ontológico", "coaching personal", etc. Respecto al concepto de coaching encontramos:

"El coaching es un camino para superar limitaciones. Permite hacer conscientes acciones, hábitos, valores, creencias, historias y juicios, a fin de facilitar procesos de cambio que permitan al cliente tomar acciones que lo lleven a ser una mejor persona, más completa. Es una oportunidad de trascender, de ir más allá. Es una manera poderosa de reinventarse a cada momento, de generar futuro, tanto a nivel personal como organizacional." (Muradep, 2012, p. 16).

Así mismo nos dice el famoso psicólogo Robert Dilts:

...es el proceso de ayuda a personas o equipos de personas a rendir al máximo de sus capacidades. Ello comporta extraer fuerzas de esas personas, ayudarlas a trascender sus barreras y limitaciones personales para alcanzar lo mejor de sí mismas, y facilitarles que puedan actuar de forma más eficaz como miembros de un equipo. (Dilts, 2004, p. 19).

Podemos leer en el Manual de Introducción al Coaching: “...es un proceso donde se ayuda a los clientes, a través de conversaciones focalizadas a crear cambios comportamentales, emocionales y cognitivos." (Vega, 2010, p. 7).

Hasta ahora observamos en estas tres definiciones ideas fundamentales como la ampliación de la autoconsciencia, el cambio o transformación personal hacia una mejor versión del sí mismo y la superación de limitaciones para el logro de objetivos dentro de contextos personales o de equipos de trabajo. Esto se logra mediante una relación interpersonal entre el coach y el "coachee" o "coacheado" donde se abordan directamente aspectos psicológicos referidos a modificar cogniciones, emociones y comportamiento. Surge entonces una pregunta crucial que es el núcleo mismo de este artículo: ¿están todas las personas que practican coaching capacitadas para ayudar a otros a lograr tales objetivos?

A partir de lo ya revisado, parece obvio que quienes deberían realizar la práctica del "coaching" son los profesionales de la salud psicológica, es decir, personas que durante varios años han estudiado a profundidad la psique humana en una universidad, incluyendo la experiencia del encuentro con otros seres humanos en las prácticas pre-profesionales. Al respecto señala Wolk:

"Para aprender a conducir un automóvil no es requisito tener conocimientos teóricos acerca 
de la combustión de un motor; en cambio, para operar en el cuerpo humano no solo basta saber blandir un bisturí. Al hacer coaching intervenimos en el ser de una persona y en su emocionalidad. El coaching está fundado en sólidas bases teóricas, rigurosas herramientas prácticas y requiere profesionales capacitados para su ejercicio". (Wolk, 2003, p. 33).

El mismo autor refiere sobre la importancia de ser profesional de la salud psicológica para la práctica del coaching: "Como psicólogo concurrente hospitalario, donde tuve enormes aprendizajes no solo profesionales sino de vida, aprendí que ponerle nombre a las cosas ayudaba muchísimo a reducir la ansiedad" (Wolk, 2003 , p. 167). Sin embargo no hay claridad respecto a esta profesionalización necesaria, dando lugar a que muchas personas que realizan esta práctica no sean profesionales de la psicología ni de la psiquiatría (medicina) (Alvarez, 2010), y es aquí donde se pone en serio riesgo la salud de quienes recurren a sus servicios.

Más adelante nos ocuparemos a profundidad de las razones por las cuales considero que la metodología llamada "coaching" es una pseudo psicología, ya que tanto su objeto de trabajo como sus procedimientos son del dominio de la ciencia humana llamada psicología y del campo de la psicoterapia. Por ahora será relevante revisar cómo es que esta práctica ha llegado a ser realizada en su mayoría por profesionales de otras áreas o incluso sin ninguna profesión (Alvarez, 2010).

\section{Una pseudo profesión posmoderna}

Actualmente y desde hace algunos años vivimos en una sociedad de consumo y orientada al éxito y a la felicidad. La tarea existencial parece ser entonces tener más y al tener más podremos ser más felices. En esta cultura posmoderna la lógica social está alterada, y se determina por la relación productores-consumidores. Todo se vende, todo se compra, todo tiene un precio (Espinoza, 2010). Lo más importante en la vida se vuelve tener un buen estatus socio económico, un buen trabajo y con esto tener los medios suficientes para tener la familia feliz, no angustiarse y no pasarla mal. Y para esto la sociedad actual empuja al hombre a tener que ser el mejor en lugar de invitarlo a dar lo mejor que tiene. Sin embargo sabemos que una vida orientada hacia el poder y el placer es una vida vacua a la que no se le encuentra sentido alguno (Frankl, 2003), porque en la búsqueda de la felicidad finalmente se descuida lo realmente valioso para la persona: su presente, sus vínculos, su trabajo, su dignidad. Sobre esta tendencia propia de la posmodernidad encontramos:

"El coaching es un juego de apuestas altas: nada menos que la felicidad. La gente acude al coaching porque quiere ser feliz o más feliz. Quiere una vida satisfactoria llena de buenas experiencias. Queremos buenas relaciones y un trabajo en el cual sentirnos orgullosos, en el cual utilizar nuestros talentos y ganar dinero. Queremos experimentar al máximo los placeres de la vida, ver nuestros buenos sueños convertidos en realidad y sentir que vivimos con nuestros valores más elevados. Queremos ser apreciados por muchos, amados por unos pocos y respetados por todos" (O'Connor y Lages, 2005, p. 30).

También es característica de esta era de éxito y felicidad la tendencia a conseguir los resultados pronto, eligiendo el camino fácil, el que menos tensiones nos acarree, el que menos malestar nos haga sentir y el que menos tiempo nos haga gastar. El objetivo existencial: resultados ya. Nos dice el célebre filósofo colombiano Estanislao Zuleta acerca de este facilismo:

"Así como se ahorra sin duda la angustia, se distribuye mágicamente la ambivalencia en un amor por lo propio y un odio por lo extraño y se produce la más grande simplificación de la vida, la más espantosa facilidad" (Zuleta, 2005, p. 14).

El facilismo, así como la búsqueda del éxito y la felicidad son las claves para comprender al fenómeno coaching, entendido como una práctica que, aunque sus defensores (sobre todo los que no son profesionales de la salud psicológica) rechacen como psicología, representa un modelo de intervención psicológica que aborda de forma directa aspectos cognitivos, emocionales y conductuales con una orientación de ayuda y mejora, bajo una visión posmoderna que considera al coach como aquel guía orientador que de alguna forma hará la vida del coachee más sencilla de vivir, más exitosa y más feliz. El psicólogo y coach Robert Dilts nos dice al respecto: 
"Guiar es el proceso de dirigir a la persona y al grupo a lo largo del camino que conduce desde el estado presente al estado deseado. Presupone que el guía ya ha estado ahí antes y conoce el camino para llegar a ese estado deseado" (Dilts, 2004, p. 29).

Para ser coach no es necesario ser profesional de la psicología ni de la psiquiatría (medicina), nos dicen las ofertas de formaciones en coaching. Cualquiera que sea mayor de edad y tenga el dinero suficiente (pues son cursos muy costosos) puede convertirse en unos cuantos meses en un "coach profesional" y trabajar con otras personas en todos esos aspectos antes mencionados. Esto es parte de la cultura en que vivimos, donde se acortan los tiempos, donde no es necesaria la ética ni la deontología, ni las regulaciones de los colegios profesionales. No, eso es muy difícil, eso no vende, la gente quiere algo corto y está dispuesta a gastar, porque quiere más. El coaching de vida, ontológico, transformacional o como queramos llamarlo, no es más que un producto de la posmodernidad dominante, una pseudo psicología disfrazada de crecimiento personal que puede poner en serio riesgo la salud de las personas. Revisemos lo que considero son las razones en que sustento esta idea.

\section{Coaching como pseudo psicología y pseudo psicoterapia}

El coaching parte de ciertas bases filosóficas y presupuestos psicológicos, tiene un objeto de estudio $\mathrm{y}$ trabajo y unos procedimientos metodológicos y técnicos para conseguir sus fines. A continuación revisaremos cada uno de estos aspectos para poder distinguir cómo es que no posee un dominio propio dentro de las ciencias por su objeto de estudio y trabajo, ni representa una alternativa de crecimiento original con metodologías y técnicas propias.

\section{Por su objeto de estudio y trabajo: ¿quiénes acuden a un coach?}

Este es un tema por demás ambiguo ya que no existe un claro límite en las competencias del coach, sobre todo del coach de vida. La idea en general es que van las personas que sin estar enfermas psíquicamente desean crecer personalmente. Nos dicen $\mathrm{O}^{\prime}$ Connor y Lages:

\begin{abstract}
"Algunas personas necesitan de un camión para moverse. Otras por ejemplo pueden sentirse satisfechas y exitosas pero creer al mismo tiempo que para mejorar no hace falta estar enferma. Estas personas contratarán a un coach para explorar con él el modo de ser más felices e incluso más eficaces. Quieren ser lo mejor que pueden ser y quieren lo mejor para ser cada vez mejores" (O'Connor y Lages, 2005, p. 29).
\end{abstract}

Sin embargo no es posible contemplar el crecimiento personal sin tomar en cuenta las restricciones psicológicas que todas las personas tenemos y que son parte de nuestra personalidad. En este sentido se vuelve imposible no trabajar con las limitaciones psíquicas que a su vez implican cogniciones, emociones, sentimientos profundos $\mathrm{y}$ comportamientos. Los mismos autores refieren lo siguiente:

"Otros problemas pueden ser más complejos y requerir varias sesiones para su resolución, como es el caso de algún quebranto significativo en la vida o de algún cambio drástico" (O’Connor y Lages, 2005, p. 28).

Resulta difícil creer que alguien que no es profesional de la salud psicológica y por ende no conoce a profundidad sobre (entre otras cosas) personalidad, emocionalidad, apego, duelo, procesos evolutivos psíquicos,motivación,psicofisiología,psicopatología y que no conoce ni tiene práctica en entrevista psicológica, observación clínica, diagnóstico psicológico ni mucho menos en metodologías psicoterapéuticas pueda realmente ayudar a quien presenta "algún quebranto significativo de la vida" y quiere sentirse mejor o "ser más feliz". Al coach acuden entonces personas que requieren de ayuda profesional:

"La emocionalidad predominante es de descontento, insatisfacción y desesperanza, y predomina la convicción de que por más que se intente hacer algo distinto, se cae siempre en el mismo círculo que obliga a repetir conductas indeseadas y a alejarse de los resultados deseados y obtenibles. Este es el momento de pedir coaching" (Muradep, 2012, p. 19). 
Hace mucho que el coaching dejó de ser sólo para deportistas y para equipos de trabajo que persiguen metas de efectividad y eficacia. El coaching es ahora un coaching de vida, existencial, que aborda temas delicados y profundos en las personas, por lo que representa un grave peligro si se realiza por quien no está adecuadamente preparado. Sobre este abordaje de "lo emocional" nos refiere la coach argentina Lidia Muradep:

"Hay estados de ánimo, como el resentimiento y la resignación, que afectan negativamente. Se manifiestan cuando nos sentimos impotentes frente a los hechos, creemos que algo es injusto, o que nos pertenece y no nos es dado, o cuando no aceptamos las situaciones que no podemos cambiar. Estos estados de ánimo nos cierran posibilidades en la vida, no nos permiten accionar y nos creemos víctimas del otro y de la situación. El resentimiento es un estado de ánimo que nos corroe el alma, nos posee, nos encadena al otro o a la situación y afecta nuestra salud, nuestras relaciones y nuestra vida" (Muradep, 2012, p. 130).

Proponiendo luego una metodología que es a simple vista psicoterapéutica:

"Esta relación tan estrecha entre lenguaje y emoción nos da la posibilidad de cambiar nuestras emociones y estados de ánimo interviniendo en el dominio del lenguaje. Las conversaciones son una herramienta de transformación emocional. Observar nuestras emociones y estados de ánimo y detectar cómo se representan a partir del lenguaje nos permite hacer una reconstrucción lingüística, es decir, cambiar la conversación o pensamiento que estamos teniendo por otra conversación o pensamiento de posibilidad, a fin de comenzar a tomar el control sobre su propia vida y manifestar cambios" (Muradep, 2012, p. 131).

En la Revista de Mentoring y Coaching se explica lo siguiente acerca del trabajo emocional y psicológico:

"Los sentimientos y las emociones son inherentes a las personas y constituyen el verdadero motivo que posterga una acción o por el que se muestra resistencia a los cambios, y es necesario que se trabajen durante todo el proceso, para obtener resultados más efectivos. Por eso, el coaching se centra, cada vez más, en las emociones que subyacen o surgen en cada cliente con diferentes manifestaciones (tristeza por los cambios, miedo a posibles fracasos, etc.) (Alvarez, 2010, p. 101).

Por ende si se desea trabajar en la motivación para el logro de metas por ejemplo, se torna necesaria una exploración existencial, psicológica y emocional respecto a las potencialidades y los limitantes para este desarrollo. Todas estas tareas son del dominio de la psicología y en especial de la psicoterapia, por lo que no representan un objeto de estudio y trabajo propio para el coaching visto como una disciplina independiente de la psicología.

Para intentar esclarecer esta ambigüedad, muchos coaches tienden a comparar el coaching con la psicoterapia para intentar deslindarlo de la psicología e independizarlo en el terreno de la ciencia y de las profesiones. En este sentido encontramos lo siguiente: "Coaching es una disciplina que resulta ser terapéutica -aun cuando este no sea su objetivo último-, mas no psicoterapéutica" (Wolk, 2003, p. 27).

¿En qué sentido resulta ser terapéutica? Y si trabaja con cognición, emoción y conducta, ¿en qué sentido no resulta ser psicoterapéutica? Sobre lo difusa que parece esa línea que separaría al coaching de la psicoterapia, Wolk refiere: "También el coaching opera en el lenguaje y -no sin sufrimiento- produce alivio y aprendizaje, aunque dolor, síntoma, inhibiciones, son tratados desde otro lugar y con otra finalidad." (Wolk, 2003 , p. 28). El mensaje resulta confuso: el coaching produce alivio y aprendizaje no sin sufrimiento, pero al mismo tiempo no se ocupa del dolor. Del dolor se ocupa la psicoterapia. La inevitable confusión tiene que ver con el hecho de que resulta imposible no contemplar el dolor humano en un proceso de transformación personal como se supone es el coaching. Por otra parte la mirada que se le suele dar a la psicoterapia para realizar la comparación y obtener las diferencias es desde el psicoanálisis ortodoxo, como lo señala por ejemplo Wolk: 
"El psicoanálisis posee una concepción singular del sujeto y del lenguaje, encontrando en la asociación libre su determinación. En este sentido, la forma en que se invita a hablar a la persona y el modo en que se lo escucha, establece también diferencias con el coaching." (Wolk, 2003, p. 28).

Pero el psicoanálisis no es por supuesto la única forma de psicoterapia ni la única forma de dialogar con el otro. El autor tendría que revisar muchas otras propuestas psicológicas y psicoterapéuticas para así intentar realizar una diferenciación. Así mismo encontramos: "Coaching no habla de enfermos, ni se ocupa de estructuras patológicas. Interviene en la dimensión de lo consciente, de la conducta observable. La visión del conflicto es diferente y está orientada hacia los resultados." (Wolk, 2003, p. 28).

Desde luego que si el autor solo se ocupa del psicoanálisis o si se basa en una perspectiva psicopatológica de la psicología (orientada desde el modelo médico reduccionista) encontrará diferencias enormes respecto al llamado coaching. Sin embargo son las mismas diferencias que se encontrarían si comparamos al psicoanálisis freudiano oal conductismo radical con la perspectiva humanista - existencial, por ejemplo. Cualquier enfoque del movimiento llamado tercera fuerza de la psicología, cuya visión de la psicoterapia no es necesariamente reducida a lo psicopatológico y cuya metodología se basa en la fenomenología, el encuentro humano y el despliegue del potencial de la persona para llegar a ser una mejor versión de sí misma (Rogers, 2011; Perls, 2002; May, 2000; Frankl, 2007), tendrá grandes similitudes con el coaching. La razón es que este ha incorporado precisamente bases filosóficas y metodologías propias del paradigma humanista existencial. Esto se debe a que casi todo el coaching de vida, ontológico, transformacional, etc., está basado en las premisas de la Programación Neuro Lingüística o P.N.L. (veremos esto más adelante al revisar la metodología).

Otro argumento utilizado por muchos coaches relacionado a este sesgo comparativo es el hecho de que el coaching no se aboca a trabajar con el pasado, con la historia, sino que se centra en el aquí y ahora y en la brecha entre la situación actual y la situación deseada (algo muy propio de la P.N.L.) Al respecto encontramos en el Manual de Introducción al Coaching:

"En Coaching nosotros partimos de esta premisa: no importa lo que haya ocurrido en el pasado, lo que no hayamos podido o no hayamos sido capaces, lo que hicimos o dejamos de hacer, lo que nos haya detenido. Nuestro poder de decisión y las herramientas con las que contamos permiten que a partir del presente definamos nuestro futuro" (Vega, 2010, p. 13).

Sin embargo la perspectiva de un ser humano posible y de intervención focalizada en el aquí y ahora hacia el proyecto es propia también de la mirada humanista - existencial, por ejemplo en la logoterapia del neuropsiquiatra austriaco Viktor Frankl, donde el proceso terapéutico está centrado en la tensión entre el ser y el llegar a ser (Frankl, 2013) o de forma similar en la teoría del psicólogo norteamericano Abraham Maslow donde la autorrealización (relacionada a la motivación, los valores, la creatividad y las metas, temas tratados en el coaching) es un tender a ser todo lo que se pueda llegar a ser (Maslow, 1991). Sin embargo es imposible como ya hemos visto, desligar la historia personal respecto a cualquier intervención psicológica aun así se "centre" en el presente, puesto que somos seres históricos con una biografía que deviene en una personalidad actual. Esto no implica considerar la historia como un determinante, pero si como un condicionante que afecta y es parte del presente personal. El coach y psicólogo argentino Leonardo Wolk en su libro Coaching, el arte de soplar brasas incluso relaciona las emociones en estricto con la historia como pasado cuando habla de indagar e investigar en las razones: "Toda emoción tiene por detrás una historia aparejada" (Wolk, 2003, p. 169).

Este autor refiere que si se trata de miedo por lo general la historia por detrás está asociada con algo malo que podría pasar; algo querido está en riesgo. $\mathrm{Si}$ se trata de enojo la mayoría de las veces la historia aparejada refiere a algo malo que pasó y no debería haber sucedido. Si se trata de la culpa por lo general la historia por detrás tiene que ver con que uno transgredió sus propios límites y provoco consecuencias no deseadas (Wolk, 2003). De esta forma podemos ver cómo tanto la visión del coaching sobre el ser humano así como su 
forma de intervención son confusas, ambiguas y poco claras, en este caso puntual, en relación a la historia y su papel en la existencia.

Vemos entonces que la estrategia del coaching para deslindarse de la psicología y la psicoterapia por su objeto de estudio y trabajo no tiene fundamento. Queda claro hasta acá que el coaching no representa una alternativa de crecimiento personal por sí misma, puesto que no trabaja ningún aspecto que no se trabaje ya de forma profesional dentro de procesos psicológicos y psicoterapéuticos. Revisemos ahora en qué sentido el coaching no representa una propuesta de crecimiento personal con un dominio propio observando su metodología.

\section{Por su metodología: el problema de la P.N.L.}

Casi todas las formas de coaching tienen su base en la Programación Neuro Lingüística (algunas utilizan también recursos de modelos como la terapia Gestalt, la Logoterapia, el Psicodrama, el Análisis Transaccional, la Terapia Cognitivo Conductual, etc.), la cual consiste en una propuesta de intervención psicológica desarrollada en los años setenta por los psicólogos Richard Bandler y John Grinder (quien era además lingüista) a partir de modelar los patrones de comunicación que utilizaban tres afamados psicoterapeutas de la época: Virginia Satir (terapia familiar sistémica), Milton Erickson (hipnoterapia) y Fritz Perls (terapia Gestalt). La forma como estos tres "magos" se comunicaban con sus consultantes era el factor clave en la transformación existencial (Bandler y Grinder, 1982). Bandler y Grinder tuvieron inicialmente una inquietud de investigación psicoterapéutica, realizando observaciones, estudios y talleres vivenciales con los terapeutas modelados, para luego ir ampliando su propuesta al terreno del aprendizaje, las organizaciones y la excelencia personal. Ellos referían no ser psicólogos, en alusión a tomar distancia de la psicología teorizante, categorial, clasificadora y prejuiciosa, donde los psicólogos son agentes promotores de iatrogenia más que de psicohigiene; de la misma forma tomaron distancia de la teología, por ejemplo. Su visión estaba en cambio teñida de una perspectiva fenomenológica, es decir, descriptiva y comprehensiva más que interpretativa y explicativa, así lo señalan los autores: "No somos psicólogos, ni teólogos, ni teóricos. No tenemos ninguna idea de la naturaleza "real" de las cosas y no estamos interesados en lo que es "verdadero". La función de modelar es llegar a una descripción que sea útil" (Bandler y Grinder, 1982, p. 17).

Probablemente esta toma de distancia respecto a la psicología como ciencia natural sea el principal argumento de quienes sin ser profesionales de la psicología o psiquiatría practican coaching con P.N.L. y tratan de diferenciarlo de la ciencia para constituirlo como un dominio propio de estudio y acción, y así no verse en la obligación ética de capacitarse estudiando psicología en una universidad acreditada ni verse regulados por ningún tipo de colegio profesional ni código deontológico relacionado a la salud.

Lo cierto es que con el tiempo la P.N.L. ha ido alcanzando a cualquiera que tenga dinero para un curso de un fin de semana con Bandler en California, prometiendo desarrollar competencias suficientes para ayudar a otras personas a ser más felices. Así se "formaron" muchísimas personas que sin ser profesionales capacitados empezaron a practicar la P.N.L., incorporándola desde los contextos deportivos y organizacionales hacia los personales, traspasando peligrosamente un límite que debe ser claro.

El coaching toma prácticamente todas las herramientas de la P.N.L. La aplicación de todos estos recursos implica un trabajo de exploración, comprensión y modificación cognitiva, emocional y conductual. Todas estas funciones y actividades son del dominio de la psicología y no representan un dominio nuevo per se. Esta base psicológica es referida en la propia literatura sobre coaching:

"La PNL nació en la década de los '70 en la Universidad de California, en Santa Cruz, Estados Unidos, donde Richard Bandler, matemático, terapeuta gestáltico y experto en informática, y el lingüista John Grinder, ambos, además, doctores en Psicología, estudiaron patrones de comportamiento comunicacional para desarrollar modelos y técnicas que pudieran explicar las estrategias de excelencia de la comunicación humana" (Muradep, 2012, p. 23).

Entre los recursos de la P.N.L. que son utilizados en el coaching y que requieren de conocimiento 
y capacitación profesional en psicología humana encontramos:

- Escucha activa y reflexiva.

- Diálogo a partir del metamodelo del lenguaje.

- Modificación de creencias limitantes.

- Detección de juicios negativos y positivos.

- Trabajo con emociones y representaciones internas.

- Preguntas poderosas que abren posibilidades.

- Cambiar la manera de ser.

- Transformación del resentimiento.

- Transformación de la resignación.

Así mismo podemos encontrar herramientas en el coaching que son propias de otras psicoterapias, las cuales revisaremos a continuación.

\section{Uso de otras metodologías psicológicas y psicoterapéuticas}

La intrusión del coaching en el campo psicológico y psicoterapéutico es amplia, como por ejemplo el hacer referencia al rapport en la relación entre coach y coachee: "El rapport es uno de los pilares fundamentales del coaching. Para establecerlo, a niveles consciente e inconsciente, es imprescindible acompasar, o sea, ir al paso del otro, sin adelantarse ni atrasarse" (Muradep, 2012, p. 70).

Así mismo podemos observar conceptos y metodologías propias de la psicoterapia cognitiva o racional emotiva:

"Sin embargo, si el cliente duda de su capacidad para alcanzar el objetivo, el coach tendrá que indagar en sus creencias. El coaching que trata con creencias es más poderoso porque no solo resuelve el problema, sino que cambia la forma de pensar que constituía ese problema" (O’Connor y Lages, 2005, p. 134).

Esta perspectiva la podemos encontrar en toda terapia cognitiva conductual o racional emotiva donde se presupone que la forma como pensamos las situaciones determinan nuestra experiencia emocional, cognitiva y conductual. La base de estos pensamientos son las creencias (Ellis y Dryden, 1989).
Incluso la coach argentina Lidia Muradep en su libro Coaching para la transformación personal ilustra en cómo utilizar un instrumento psicológico al cual llama "Formulario para la identificación de creencias limitadoras" (Muradep, 2012, p. 141). Así mismo refiere la forma como cambiar las creencias del cliente a través del proceso de cambio de creencias (Muradep, 2012, p. 148). A simple vista aspectos que se trabajan en ambientes psicoterapéuticos facilitados por un profesional de la psicología capacitado y formado en psicoterapia y que representan un grave riesgo para la salud en caso sean abordados por quienes no tengan el adecuado estudio y formación.

También podemos encontrar recursos psicodramáticos en esta práctica. Así lo señala Wolk cuando habla de "las técnicas psicodramáticas en el proceso de coaching" (Wolk, 2003 p. 185), incluso refiere lo sensible que puede resultar este trabajo:

"Consideramos imprescindible un contexto de confianza y legitimación para abordar el trabajo con técnicas dramáticas. Esta forma de intervención requiere un aprendizaje profundo y el cuidado de la seguridad psicológica. Sobre todo al comienzo, cuando se movilizan muchas ansiedades relacionadas con el mirar y ser mirado por otros, verse, no ser visto o reconocido, exponerse a una evaluación, a los sentimientos negativos de otros, poner el cuerpo, desempeñar roles rechazados o de personajes repudiables, al ridículo, al descontrol, a equivocarse, a lo nuevo, a la expresión de los afectos, a lo erótico, al amor, al rechazo, al cambio, al darse cuenta, etc." (Wolk, 2003, p. 186).

La necesidad de ser profesional de la salud psicológica para realizar estas funciones resulta clara, puesto que son metodologías que exploran e inciden sobre las emociones, el pensamiento y la conducta con fines de cambio, siendo este un propósito relacionado a la salud. La práctica del coaching por quienes no son profesionales de la psicología constituye un grave riesgo al no tener los conocimientos, la capacitación y la experiencia necesarios para ayudar como la ética lo exige. De aquí se desprende otro aspecto importante que caracteriza peligrosamente al coaching: la falta de regulación. 


\section{Ausencia de regulación}

Ningún coach puede ser regulado por institución alguna que esté basada en una ética profesional y regida por criterios deontológicos puesto que el coaching no está comprendido dentro de la psicología como ciencia humana y de la salud. No existe en las universidades más que como cursos de extensión o posgrados breves. No existe un "colegio de coaches" que regule la práctica de sus colegiados. Solo existen asociaciones, federaciones y un sin número de instituciones que plantean sus propios criterios éticos pero sin tener un fundamento académico ni científico. El coaching reclama formalidad y ser una ciencia con dominio propio pero al mismo tiempo guarda distancia de lo formal, de lo serio, de lo que cuesta, y por lo tanto está lejos de ser aceptado como ciencia o como una profesión con un campo propio. $\mathrm{Al}$ respecto podemos leer:

\begin{abstract}
"Algunos expertos consideran que todavía no puede considerarse una profesión ya que carece de un cuerpo normalizado de conocimiento que sirva para guiar la práctica de coaching. Existen un conjunto de normas deontológicas dentro de las asociaciones de coaching, pero sería necesario que existieran protocolos de actuación que permitieran estandarizar los procesos, ya que aunque existen algunas pautas "universales", las distintas escuelas presentan enfoques y prácticas muy distintos. Es importante señalar que, en la Clasificación Nacional de las Ocupaciones (2002) no está incluida la profesión de coach y tampoco en el borrador que se está preparando para 2010. También parece necesario llegar a una definición del concepto más unívoca y profundizar en el propio término, desde el punto de vista académico e investigador" (Alonso, Calles y Sánchez, 2010, p.94).
\end{abstract}

De esta formael ejercicio de funciones o actividades propias del psicólogo se ve usurpado por quienes no son profesionales de la salud psicológica, siendo este un grave peligro para la salud y por supuesto una grave falta a la ética y a todo criterio deontológico. Así lo señala el Código de Ética del Colegio de Psicólogos del Perú:

"Art. 120.- Constituye grave infracción del Código de Ética Profesional y será sancionado conforme a las correspondientes disposiciones del Reglamento interno y el Estatuto del Colegio de Psicólogos del Perú, sin perjuicio de las sanciones penales por ejercicio ilegal de la profesión:

c) Toda persona que ejerza funciones o actividades de psicólogo sin tener el titulo correspondiente, aunque posea títulos o grados de otra profesión;

d) Toda persona que, sin tener título alguno, ejerza funciones o actividades de psicólogo" (Colegio de Psicólogos del Perú, 2011, p. 23).

Entendiendo como funciones del psicólogo:

"Art. 3.- Su actividad se orienta a la identificación, promoción, desarrollo y rehabilitación de las funciones psicológicas que aseguren un adecuado desarrollo humano de todas las personas en el ámbito educativo, de salud, laboral, sociocultural, económico, recreativo y político." (Ley de Trabajo del Psicólogo, 2004, p. 279255).

Siendo así el coaching resulta en una pseudo psicología sin campo de estudio y trabajo propios y sin teorías, metodologías ni técnicas independientes del dominio de la psicología y la psicoterapia. Por lo tanto debe considerarse su práctica como un peligro para la salud de las personas en caso sea realizada por quienes no son profesionales de la psicología o psiquiatría y sin formación alguna en psicoterapia. En caso sea realizada por un profesional de la salud psicológica, le haría la invitación a llamarse a si mismo psicólogo y no coach.

\section{Una propuesta a la crisis posmoderna: esbozo de una cultura psicohigiénica}

Hay que aceptar que parte de esta crisis tiene que ver con la pasividad de los psicólogos y los colegios profesionales frente a las necesidades actuales de las personas, permitiendo que se considere a la psicología como una ciencia natural propia del siglo XIX.La única forma de hacer frente a los embates de la posmodernidad es desarrollando una perspectiva psicohigiénica de la psicología y la psicoterapia, es decir, una visión de la salud psicológica que no se reduzca al tratamiento de la psicopatología (rehabilitación) sino que integre además el desarrollo de los recursos y potencialidades de todas las personas (identificación, promoción y desarrollo). Es necesario ampliar la noción de psicología como 
ciencia humana y no solo natural, y que contempla no solo la restricción sino el crecimiento personal desde diversas aristas, como el margen de libertad, la responsabilidad, los valores y la potencialidad inherente a cada ser humano. El viejo cliché (que parecía ya superado) que refiere que "al psicólogo van solamente los locos" está volviendo, alimentado por quienes no tienen la suficiente vocación para formarse como la ética lo presupone, e impulsado por quienes (incluso siendo profesionales de la salud psicológica) realizan cursos abiertos a todo público, porque hay demanda, porque está de moda ser coach, y porque vende.

Una cultura psicohigiénica también busca educar en responsabilidad a las personas, invitándolas a ser más conscientes de la importancia de cuidar su salud psicológica emocional. Investigar previamente acerca del perfil profesional del facilitador o coach, tomando en cuenta que cualquier actividad considerada de ayuda y que involucre una relación interpersonal donde se trabaje directamente sobre los pensamientos, creencias, emociones y sentimientos buscando cambios en la conducta ha de ser realizada por un profesional de la salud psicológica con formación en psicoterapia. Ahí están los medios de comunicación, las redes sociales, los auditorios, los salones de clase para difundir, para prevenir y para promocionar estilos de vida psicológicamente más saludables.

Es tarea de todos y especialmente de los profesionales de la salud psicológica, la constitución de esta cultura psicohigiénica, como un esfuerzo por privilegiar la salud y la dignidad humana frente a los condicionamientos que la posmodernidad nos impone.

\section{REFERENCIAS}

Alonso, M., Calles, A. y Sánchez, C. (2010) Desarrollo de personas y de las organizaciones coaching ejecutivo y Mentoring. Conceptos. En Revista Mentoring y Coaching (3). España: Universidad Politécnica de Madrid y Universidad Complutense de Madrid.

Fecha de recepción: 28 de marzo, 2018

Fecha de aceptación: 17 de mayo, 2018
Alvarez,A. (2010) Por y hacia dónde camina el coaching. En Revista Mentoring y Coaching (3). España: Universidad Politécnica de Madrid y Universidad Complutense de Madrid.

Bandler, R. y Grinder, J. (1982) De sapos a príncipes. Chile: Cuatro vientos.

Colegio de Psicólogos del Perú (2011) Código de ética. Perú: Colegio de Psicólogos del Perú.

Dilts, R. (2004) Coaching, herramientas para el cambio. España: Urano

Ellis, A. y Dryden, W. (1989) Práctica de la Terapia racional emotiva. España: Desclée de Brouwer.

Espinoza, L. (2010) La posmodernidad, lo sublime y un ojo cortado. En Poliantea 6 (11). Colombia: Politécnico Grancolombiano.

Frankl, V. (2013). Psicoanálisis y existencialismo. México: FCE.

Frankl, V. (2007). Logoterapia y análisis existencial. España: Herder.

Frankl, V. (2003). Ante el vacío existencial. España: Herder.

Maslow, A. (1991) Motivación y personalidad. España: Díaz de Santos editores.

May, R. (2000). El Dilema del hombre. Colombia: Gedisa.

Ministerio de Trabajo (2004) Ley de trabajo del psicólogo. En El Peruano. Perú: Editora Perú.

Muradep, L. (2012) Coaching para la transformación personal. Argentina: Granica

O’Connor, J. y Lages. A. (2005) Coaching con PNL. España: Urano

Perls, F. (2002) Sueños y existencia. Chile: Cuatro Vientos

Rogers, C. (2011) El proceso de convertirse en persona. España: Paidos Ibérica.

Vega,K. (2010) Curso de introducción al coaching. Manual de apoyo. España: International Coaching Group.

Wolk, L. (2003) Coaching, el arte de soplar brasas. Argentina: Gran Aldea Editores

Zuleta, E. (2005) Elogio de la dificultad y otros ensayos. Colombia: Hombre nuevo editores. 